Meta

Journal des traducteurs

Translators' Journal

\title{
Langue, langage et les étonnantes locutions, expressions et phrasèmes du français, et leurs correspondants en anglais et en allemand
}

\section{André Clas}

Volume 60, numéro 2, août 2015

$60^{\mathrm{e}}$ anniversaire. Les horizons de la traduction : retour vers le futur $60^{\text {th }}$ Anniversary. Translation's Horizons: Back to the Future

$60 \mathrm{mo}$ aniversario. Los horizontes de la traducción: regreso al futuro

URI : https://id.erudit.org/iderudit/1032859ar

DOI : https://doi.org/10.7202/1032859ar

Aller au sommaire du numéro

Éditeur(s)

Les Presses de l’Université de Montréal

ISSN

0026-0452 (imprimé)

1492-1421 (numérique)

Découvrir la revue

Citer cet article

Clas, A. (2015). Langue, langage et les étonnantes locutions, expressions et phrasèmes du français, et leurs correspondants en anglais et en allemand. Meta, 60(2), 299-305. https://doi.org/10.7202/1032859ar
Résumé de l'article

La présente étude est une comparaison raisonnée de locutions, expressions et phrasèmes du français, somme toute assez étonnantes, et de leurs correspondants en anglais et en allemand. Elle illustre le fait que la langue donne une structuration au flou, c'est-à-dire qu'elle crée son existence par concepts. En fin de compte, la traduction est la reproduction du pensable d'une langue dans une autre langue. 


\title{
Langue, langage et les étonnantes locutions, expressions et phrasèmes du français, et leurs correspondants en anglais et en allemand
}

\author{
André Clas \\ Université de Montréal, Montréal, Canada \\ andre.clas@gmail.com
}

\begin{abstract}
RÉSUMÉ
La présente étude est une comparaison raisonnée de locutions, expressions et phrasèmes du français, somme toute assez étonnantes, et de leurs correspondants en anglais et en allemand. Elle illustre le fait que la langue donne une structuration au flou, c'est-à-dire qu'elle crée son existence par concepts. En fin de compte, la traduction est la reproduction du pensable d'une langue dans une autre langue.
\end{abstract}

\section{ABSTRACT}

The present study offers a reasoned comparison of rather astonishing French locutions, expressions, and idioms, and their English and German equivalents. It shows how language structures the haziness of experience, that is to say language creates its own existence through concepts. In sum, translation is the reproduction of the thinkable world of one language into another.

\section{MOTS-CLÉS/KEYWORDS}

langue, langage, locutions, expressions, phrasèmes

languages, language, locutions, expressions, idioms

Et une réalité pour nous est quelque chose qui nous est connu et qui par conséquent n'appartient pas exclusivement au monde extérieur.

(Hjelmslev 1971: 193)

Comme on le sait, une langue est un système de communication qui assure l'intercompréhension entre les individus qui utilisent ce même système, mais il faut sans doute ajouter que ce système est également et surtout une entité qui repose sur les fonctions intellectuelles et assure ainsi la mémoire et la pensée, soit le connu de l'expérience passée et le développement de l'avenir. En fait, on peut dire qu'une langue est un outil de communication qui s'appuie sur le réservoir de symboles créés par l'esprit et qui sont reconnus par la communauté des locuteurs, c'est-à-dire que le langage est également un développement social qui assure l'existence, incorpore les changements, les modifications et les créations; en fait une langue est un outil en constante innovation qui est au service des usagers et répond ainsi à leurs besoins. Ces nécessités qui peuvent être fort variables et qui vont de l'abandon de l'actuel en linguistique au changement et à l'innovation selon divers procédés acceptant la créativité soumise à une matrice, à la transformation de cette matrice, à l'emprunt à une autre langue ou à l'influence d'un dialecte, au calque de l'emprunt d'une autre langue. Bien entendu l'aspect social des niveaux de langue joue également un rôle important 
puisque l'on peut y distinguer des niveaux recherchés, familiers, populaires et argotiques. L'ensemble est soumis en règle générale à une adaptation phonétique de la langue emprunteuse qui peut transformer la prononciation et la graphie de l'emprunt. Comme les mots sont en fait des symboles, ils n'ont pas un sens intrinsèque, mais un référent ou une désignation; c'est bien entendu la porte ouverte à de nombreuses innovations sémantiques et des créations selon la perception des usagers et selon les influences liées aux caractères ludiques de la société.

Les exemples ne manquent pas, il suffit de parcourir les dictionnaires étymologiques. À titre d'illustration, on peut citer ovation qui selon Le Petit Robert ${ }^{1}$ est emprunté au latin ovatio, de ovis (brebis) et qui désignait une "cérémonie en l'honneur d'un général victorieux accompagnée d'un sacrifice d'une brebis» (p. 1335). De nos jours, il s'agit tout simplement d'une acclamation publique. On peut encore rappeler que hécatombe est tiré du grec signifiant «sacrifice de cent bœufs» qui maintenant indique «un massacre d'un grand nombre d'hommes» (p. 918), et aussi montrer que si cheval provient du latin caballus, les adaptations phonétiques sont remarquables, tout comme bigot et bigotte dérivent de l'anglais by God. On sait que pour rendre par exemple l'allemand Bücherwurm ou l'anglais bookworm le français transforme le ver du livre allemand ou anglais en rat: rat de bibliothèque. Il est vrai qu'on pourrait ici rappeler l'extrait de Paul Valéry:

Le Langage est étourdi - oublieux. Les significations successives d'un mot s'ignorent. Elles dérivent par des associations sans mémoire et la troisième ignore la première. (Valéry 1960: 621, cité dans L'insolite. Dictionnaire des mots sauvages², p. 325).

Comment ne pas alors affirmer que les langues sont surtout formées d'expressions idiomatiques puisque chaque langue a ses particularités phonétiques et ses spécificités orthographiques et grammaticales, et bien sûr un agencement mental par un choix de symboles uniques. Comme chacun le sait, la traduction d'une langue à l'autre est bien entendu possible puisqu'on ne traduit pas des mots mais la signification dégagée du référent du signe. Il en va de même pour les expressions, phrasèmes et pragmatèmes. Dans ces cas la substitution est un changement de symbole déterminé par la communauté sociale. Les expressions idiomatiques se forment par des métaphores qui ont des particularités linguistiques propres à chaque langue et qui ont souvent une particularité phonétique, soit une sonorité spécifique, un rythme particulier, une alternance de sons. Ainsi, l'allemand Morgenstund hat Gold im Mund [l'heure matinale a de l'or en bouche] a un rythme particulier et une alternance vocalique qui ne se retrouve pas, ni dans l'équivalent anglais It's the early bird who gets the worm [c'est l'oiseau du matin qui attrape le ver], ni dans l'équivalent français La fortune sourit au lève tôt. Ajoutons qu'il y a également une variation de niveau de langue entre les trois expressions, mais le parallélisme reste valable, car il n'y a aucune autre solution puisque les mots d'une langue ne correspondent pas à ceux d'une autre et chaque expression garde la même valeur.

Les dénominations des entités du réel et du pensé sont existentielles par la pensée et dénotent un sens déterminé par la formation d'une signification d'un concept. En fait, la pensée peut être conçue comme donnant au pensable et aux choses appartenant à la réalité existentielle linguistique. La langue donne ainsi une structuration au flou, c'est-à-dire crée son existence par concepts. La traduction est en fait la reproduction du pensable d'une langue dans une autre langue. 
Examinons quelques exemples d'expressions en français tirés du dictionnaire $L e$ Petit Robert en donnant les homologues anglais et allemands expliqués.

(1) Abattre son jeu; déposer ses cartes sur la table [faire connaître ce que l'on veut faire]

Cette expression correspond exactement aux expressions anglaise to lay one's cards on the table et allemande seine Karten aufdecken.

(2) Les chiens aboient, la caravane passe [ne pas s'occuper des envieux qui crient]

a comme équivalent anglais : sticks and stones may brake my bones (but words will never hurt me (Hachette-Oxford ${ }^{3}$ ) et allemand: man soll sich durch Kritiken und böses Gerede nicht beirren lassen (Sachs-Villatte ${ }^{4}$ ). Dans ce cas, l'anglais explicite l'expression en disant qu'on peut se casser les os, mais que les paroles ne feront jamais de mal et l'allemand précise qu'on ne doit pas se laisser déconcerter par des critiques et des paroles méchantes. Dans les deux cas, il s'agit d'une explicitation qui rend compte de la signification de l'expression.

(3) Ce n'est pas un aigle [ne pas être d'une intelligence supérieure]

correspond à l'anglais not to be the brightest ou to be no genius (Larousse ${ }^{5}$ ), et à l'allemand: er, sie ist kein großes (Kirchen)Licht, soit il ou elle n'est pas une grande lumière.

(4) Être gonflé à bloc [être plein d'ardeur]

a comme équivalents en anglais: to be raring to go (Hachette-Oxford), soit être très impatient de faire qch, et allemand draufgängerisch (Sachs-Villatte), soit qui risque tout, qui va droit au but.

(5) Aller, poursuivre son petit bonhomme de chemin [poursuivre sans hâte ce qu'on est en train de faire]

donne en anglais: to jog on, et en allemand: ruhig, unbeirrt seinen Weg gehen, sein Ziel verfolgen (Sachs-Villatte), c'est-à-dire aller tranquillement, sans se laisser détourner de son chemin et en poursuivant son but.

(6) Être triste comme un bonnet de nuit [personne triste et ennuyeuse]

qui donne en anglais: to be as boring as hell (Hachette-Oxford), soit être aussi ennuyeux que l'enfer, et en allemand: ein grämiger Mensch sein, soit être un individu grognon, grincheux.

(7) Rire comme un bossu [rire à gorge déployée]

se traduit en anglais : to laugh like a drain (Hachette-Oxford) ou to laugh fit to bust (Larousse), c'est-à-dire rire comme un drain ou rire prêt à éclater, et en allemand: sich krumm und schief lachen, à se tordre et se pencher de rire.

(8) En avoir plein les bottes, ou le dos, ou le cul [en avoir à satiété, ras-le-bol] 
se traduisent en anglais par: to be fed up (to the back teeth) (Oxford-Hachette) ou to be sick to death (Larousse), soit être nourri jusqu'aux molaires ou être malade à en mourir, et en allemand: die Nase voll haben, en avoir plein le nez.

(9) Les bras m'en tombent [être stupéfait]

correspond à l'anglais: I'm speechless (Hachette-Oxford), soit rester muet, et à allemand: da bin ich sprachloss, soit également rester muet.

(10) Compter pour du beurre [ne pas compter]

se traduit en anglais: to count for nothing (Oxford-Hachette), en allemand: ùberhaupt nicht zählen. L'anglais et l'allemand traduisent la signification de l'expression.

(11) Vouloir le beurre et l'argent du beurre [vouloir tout avoir]

correspond à l'anglais: to want to have one's cake and eat it, vouloir avoir à la fois son gâteau et le manger. Il n'y avait aucune traduction allemande dans les dictionnaires (proposition: er, sie will den Kuchen haben und auch essen).

(12) Être comme cul et chemise [être inséparables]

a comme équivalent anglais: to be as thick as thieves (Robert \& Collins; Larousse), soit être liés comme des voleurs, explicitation qui correspond également à s'entendre comme larrons en foire, et allemand: unzertrennlich sein (Sachs-Villatte), soit être inséparables.

(13) Monter sur ses grands chevaux

[s'emporter, le prendre de haut]

se traduit en anglais par: to get on one's high horse (Hachette-Oxford), et en allemand par: aufbrausen, hochgehen (Sachs-Villatte), soit s'emporter, s'emballer, se mettre en colère. L'anglais a une traduction littérale et l'allemand explicite la signification de l'expression.

(14) Ménager la chèvre et le chou [ne pas prendre parti, être opportuniste]

correspond à l'anglais: to sit on the fence (Hachette-Oxford), soit être assis sur la clôture; to run with the hare and hunt with the hounds (Larousse), soit courir avec le lièvre et chasser avec les chiens, en fait miser sur les deux tableaux (HachetteOxford); et à l'allemand: es mit keinem verderben wollen, soit ne pas vouloir gâter les relations avec personne. Ici également, c'est la signification et non la métaphore qui est le choix.

(15) Garder à qn un chien de sa chienne

[lui garder rancune, lui préparer une vengeance]

correspond à l'anglais: to bear a grudge against sb (Hachette-Oxford), soit avoir de la rancune contre qn, ou encore to have a rod in pickle for s.o. et I'll get even with sb (Robert-Collins), respectivement avoir une baguette en conserve pour qn et se venger de qn. L'expression correspond aussi à allemand: das werde ich ihm heimza- 
hlen; das soll er mir büßen, soit il va me le payer; tu me le paieras. Toutes les diverses possibilités pour rendre le phrasème s'appuient sur la signification.

(16) Se faire rouler dans la farine [duper qn, se faire avoir, voler qn]

correspond à l'anglais: he was had (Robert-Collins), to be had (Hachette-Oxford); soit se faire avoir, il a été attrapé, et à l'allemand: jemanden hereinlegen, soit littéralement mettre qn dedans c'est-à-dire attraper, avoir, posséder, blouser qn. Les équivalents anglais et allemand correspondent à la signification du phrasème originel mais la métaphore française est perdue.

(17) Il n'a pas inventé le fil à couper le beurre

[il n'est pas bien malin]

correspond à l'anglais: he isn't the sharpest knife in the drawer : il n'est pas le couteau le plus acéré dans le tiroir; et à allemand: er hat das Pulver nicht erfunden (Langenscheidts): il n'a pas inventé la poudre; et en français du Québec: il n'a pas inventé le bouton à quatre trous. Les équivalents de ce phrasème correspondent à des locutions différentes.

(18) Courir plusieurs lièvres à la fois; courir deux lièvres à la fois [mener plusieurs activités en même temps]

correspond en anglais à : to have several irons in the fire (Hachette-Oxford); to try to keep two pots on the boil (Robert-Collins); to try to do two things at once (Larousse); soit avoir plusieurs fers au feu; essayer de garder deux pots en ébullition; essayer de faire deux choses à la fois; et à l'allemand: zwei Dinge auf einmal betreiben; zwei Ziele auf einmal verfolgen; soit faire deux choses à la fois; poursuivre deux buts ensemble. La traduction de cette double expression est variable, dans la première équivalence il y a le remplacement par fer, ce qui correspond comme les autres équivalences à la signification conceptuelle tout comme l'allemand, c'est-à-dire on a fait un choix dans le domaine des possibilités.

(19) Être dans la lune

[être très distrait, hors de la réalité]

correspond à l'anglais: to have one's head in the clouds (Hachette-Oxford); to be far away (Larousse); soit littéralement avoir la tête dans les nuages; être très loin; et en allemand: nicht bei der Sache sein; geistesabwesend sein; mit seinen Gedanken ganz woanders sein (Langenscheidt), soit ne pas être présent; avoir l'esprit absent; être ailleurs dans ses pensées ce qui correspond parfaitement à la signification du phrasème originel.

(20) Ne pas mâcher ses mots; ne pas y aller par quatre chemins

[s'exprimer avec une franchise brutale, dire tout $\mathrm{cru}$ ]

correspond à l'anglais: not to mince one's words, soit ne pas hacher ses mots, et à l'allemand: kein Blatt vor den Mund nehmen; frei von der Leber weg sprechen (Langenscheidt), soit littéralement ne pas prendre de feuille devant la bouche; parler librement et franchement du foie (= à cœur ouvert). Ici encore avec une métaphore différente en allemand, on retrouve la signification du phrasème originel.

(21) Passer de la pommade à qn; lécher le cul à qn

[flatter servilement] 
correspond à l'anglais : to butter sb up, to soft-soap sb (Robert-Collins), soit littéralement beurrer qn, savonner avec douceur qn; et à l'allemand: jemandem schmeicheln, schöntun, um den Bart gehen; Hönig um den Mund, Bart schmieren (Langenscheidt) soit flatter qn, minauder, tourner autour de la barbe, mettre du miel autour de la bouche, de la barbe. Les homologues anglais et allemands correspondent à la signification du phrasème originel.

(22) Pleurer comme une Madeleine, comme une vache, comme un veau, à chaudes larmes [pleurer abondamment]

Tous ces phrasèmes sont synonymes et correspondent à l'anglais: to cry one's eyes out, soit littéralement pleurer à en sortir les yeux; et en allemand: heulen wie ein Schloßhund, soit: pleurer comme un chien de château. Si l'anglais intensifie l'expression par pleurer jusqu'à perdre les yeux, l'allemand qui a deux verbes pour pleurer: heulen et weinen, exprime l'intensité des pleurs et des hurlements par heulen et par une nouvelle image.

(23) Tomber dans les pommes

[s'évanouir]

correspond à l'anglais: to pass out, to faint, et à l'allemand: ohnmächtig werden, in Ohnmacht fallen, umkippen (Langenscheidt), soit s'évanouir, tomber en évanouissement. Les correspondants en anglais et en allemand correspondent à la signification du phrasème. Il ne pouvait en être autrement comme le signale Guiraud (1961: 78), puisque «l'expression remonte à tomber dans les pasmes, ancien substantif déverbal de se pâmer: l'expression signifie donc tomber en pâmoison». Il s'agit donc d'une confusion phonétique historique et la métaphore est intraduisible.

(24) Quant les poules auront des dents [jamais]

correspond à l'anglais: when pigs can fly, have wings (Robert-Collins), soit quand les cochons pourront voler ou avoir des ailes; et à l'allemand: wenn Ostern und Pfingsten auf einenTag fallen (Langenscheidt), soit quand Pâques et la Pentecôte tomberont sur le même jour. Nous avons ici une différence totale de la façon d'illustrer la même signification impossible de se réaliser.

(25) Engueuler qn comme du poisson pourri

[exprimer fortement son mécontentement à qn]

correspond à l'anglais: to call sb all names under the sun, to bawl at sb (RobertCollins), soit appeler qn par tous les noms sous le soleil, engueuler qn; et à l'allemand: jemanden anscheißen, zur Sau machen (Langenscheidt), soit chier sur qn, traiter qn de truie. Naturellement cette locution dans une langue fortement populaire est délicate à traduire, mais la signification en anglais et en allemand correspond bien à la langue source même si le sens se moule d'une autre façon.

Tous ces exemples montrent bien que les expressions ou phrasèmes des langues ne peuvent que très rarement passer telles quelles d'une langue à une autre. La métaphore est soit remplacée par une autre, d'autant plus qu'il s'agit d'exemples de niveaux de langues populaires ou familières. Les langues, comme on le sait, sont des expressions des représentations des visions du monde et sont donc forcément multiples et diverses comme le prouvent les exemples cités ou encore comme on peut le voir 
facilement avec les exemples suivants: l'anglais to have a hangover se dit en français avoir la gueule de bois et en allemand einen Kater haben (avoir un chat mâle). En fait, c'est une illustration de la même réalité mais dont l'expression linguistique de la pensée se présente de façon différente et par conséquent comme chaque mot d'une langue invoque d'autres mots de la langue les résultats sont naturellement différents. Il faut y ajouter encore que les langues sont des phénomènes liés à l'histoire et donc également à une évolution différente l'une de l'autre, ce qui explique d'ailleurs la nécessité pour toutes de changement par des néologismes ou des calques et emprunts. Aucune langue, on le sait, ne se suffit à elle-même et chacune détermine la réalité par la pensée qui donne une connaissance des choses et donc un concept avec une dénomination et c'est cette signification qu'on traduit dans l'autre langue. Comme le signalait Bruno Liebbrucks (1964:75) : "Sprache ist Brücke nicht zwischen Ländern, sondern zwischen Abstraktionen. Sie ist das einzige menschliche Land», c'est-à-dire la langue est un pont non pas entre les pays mais entre les abstractions. Elle est l'unique pays humain.

\section{NOTES}

1. Le Petit Robert (1989): Paris: Dictionnaires Le Robert.

2. Rheims, Maurice (1989): L’insolite. Dictionnaire des mots sauvages. Paris: Larousse.

3. Le Dictionnaire Hachette-Oxford (1994): Oxford: Oxford University Press.

4. Langenscheidts Grosswörterbuch (1979): Berlin: Langenscheidt.

5. Dictionnaire français-anglais de locutions et expressions verbales (1973): Paris: Larousse.

\section{RÉFÉRENCES}

Gónzalez Royo, Carmen et Mogorrón Huerta, Pedro (2011): Fraseología contrastiva: lexicografía, traducción y análisis de corpus. Alicante: Publicaciones de la Universidad de Alicante. Guiraud, Pierre (1961): Les locutions françaises. Que sais-je? (nº 903). Paris: PUF.

Hjelmslev, Louis (1971): Prolégomènes à une théorie du langage. Paris: Les Éditions de Minuit. Liebrucks, Bruno (1964): Sprache und Bewußtsein. Frankfurt am Main: Akademische Verlagsgesellschaft.

Lyons, John (1970): Linguistique générale. Paris: Larousse.

Martin, Robert (2001): Sémantique et automate. Paris: PUF.

Mejri, Salah, Gross, Gaston, Clas, André et Baccouche, Taïeb (1998): Le figement lexical. Actes de la $1^{r e}$ Rencontre Linguistiques Méditerranéennes. Tunis: Cérès éditions.

Pariente, Jean-Claude (1969): Essais sur le langage. Paris: Les Éditions de Minuit.

Wierzbicka, Anna (1992): Semantics, Culture and Cognition. Oxford: Oxford University Press. WiLls, Wolfram (1992): Übersetzungsfertigkeit. Tübingen: Gunter Narr Verlag.

Ph.D. Doktor der Philosophie de l'Eberhardt-Karl Universität, Tübingen, André Clas est Professeur émérite de l'Université de Montréal, Docteur Honoris Causa de I'Université d'Alicante, Chevalier des palmes académiques (France), Membre de la Société royale du Canada, Membre d'honneur de la Société des traducteurs du Québec et récipiendaire de la Médaille Joseph Zaarour, décernée par l'ÉTIB (Université Saint-Joseph de Beyrouth). II est en outre le fondateur de la Banque de Terminologie (TERMIUM) et du réseau LTT (Lexicologie, traduction, terminologie, AUPELF-UREF). II a dirigé le Groupe de recherche en sémantique, lexicologie et terminologie (GRESLET) et la revue META, Journal des Traducteurs de 1967 à 2007. Lors des célébrations de son $125^{\mathrm{e}}$ anniversaire, l'Université de Montréal lui a accordé le titre de «Pionnier» pour souligner l'excellence de sa contribution à la renommée de l'institution. 